\title{
Gingival abscess occurring in maxillary primary central incisor fused to supernumerary tooth
}

\author{
Naofumi Kamakura, Kazuhiko Nakano, Kayoko Nagayama, Ayuchi Kojima, \\ Yukiko Takashima, Michiyo Matsumoto and Takashi Ooshima \\ Department of Pediatric Dentistry, Osaka University Graduate School of Dentistry \\ 1-8 Yamada-oka, Suita, Osaka 565-0871, JAPAN
}

\begin{abstract}
A 5Y7M-old girl was referred to our clinic by a general dentist for detailed examination of a recurrent gingival abscess in the maxillary left primary central incisor region. An intraoral examination revealed a diffuse swelling in the palatal gingiva area of the affected tooth. A periapical radiograph showed that the affected tooth was actually the primary central incisor and a supernumerary tooth, which had fused and been filled with composite resin at the juncture of the 2 tooth crowns. Removal of the filling induced severe bleeding and root canal treatment was performed. Radiographic examinations revealed 2 independent root canals, thus an additional access cavity to the mesial pulp cavity was produced for further root canal treatment. After the lesion had disappeared, root canal filling was performed using iodoform-calcium hydroxide paste and the affected tooth was restored with composite resin to fashion a single crown. However, the canal filling paste was later observed to be resorbed in the cervical area of the root. At $6 \mathrm{YOM}$, the patient received a traumatic injury in the anterior teeth region and the fused tooth was drastically displaced in a palatal direction, thus we extracted it under infiltration anesthesia. The root was observed to be wide in the mediodistal direction, and a histopathological examination revealed only dentin between 2 independent root canals. Our findings showed that the fused tooth was composed of 2 crowns and 1 root with 2 independent canals, and indicate that each crown and canal should have been treated separately.
\end{abstract}

\author{
Key words \\ Fused tooth, \\ Gingival abscess, \\ Primary tooth, \\ Supernumerary tooth, \\ Trauma
}

\section{Introduction}

Tooth fusion is defined as a union between the dentin and enamel of 2 or more separate developing teeth, with appearance of the partial and total types considered to be dependent on tooth development stage at the time of union ${ }^{1}$. The incidence of this anomaly in Caucasians is reported to be approximately $0.1 \%$ in permanent dentition and $0.6 \%$ in primary dentition $^{2}$. On the other hand, the frequency of fused teeth in Japanese is higher, as analyses of 4,422 subjects in Japan aged 3-6 years old revealed a detection frequency of $2.8 \%{ }^{3)}$, among which approx-

Received on September 30, 2009

Accepted on January 25, 2010 imately four-fifths of the cases were found in the mandible, with the incisors and canines the most frequently affected ${ }^{3,4)}$. Although tooth fusion generally occurs between 2 adjacent teeth, cases involving supernumerary teeth are extremely rare in primary dentition, with an estimated prevalence of $0.06 \%$ in Japanese children, and $0.2-0.6 \%$ in children in Western countries and China ${ }^{5}$.

A fused tooth with 2 separate root canals and a single wide crown is a common finding, though separation into 2 single crowns has been reported ${ }^{6}$. Typical problems associated with fused teeth include esthetics, caries, periodontal disease, and malocclu$\operatorname{sion}^{7)}$. Treatment strategies have been rarely reported, though it appears to be difficult to treat such teeth, especially with endodontic therapy, due to the 

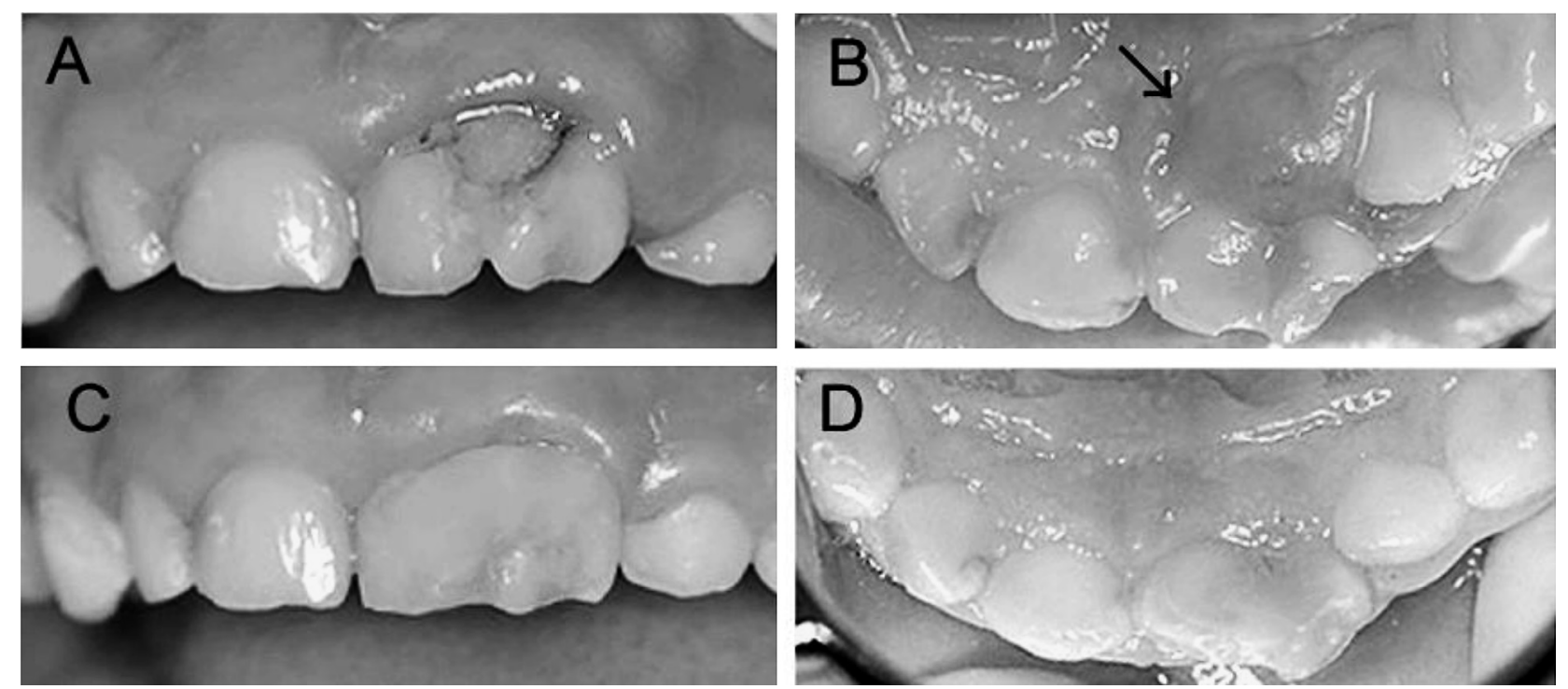

Fig. 1 Intraoral photographs

Images shown were obtained prior to treatment (A and B) and 54 days after the first visit (C and D). Labial view: A and C. Palatal view: B and D.
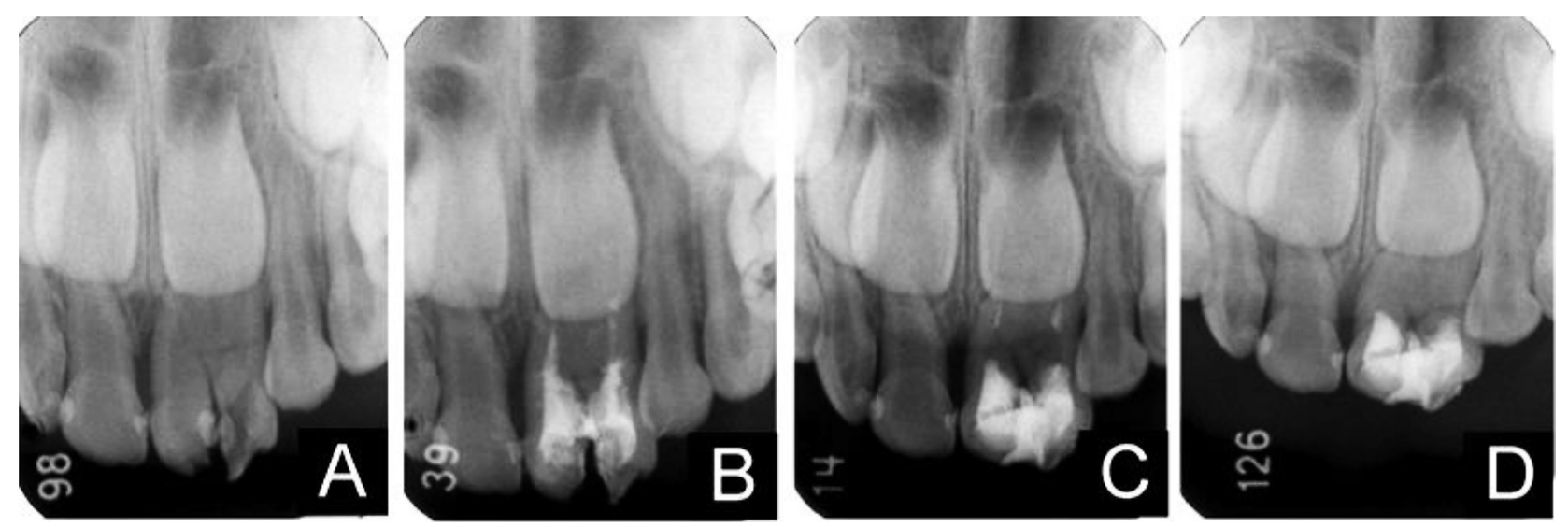

Fig. 2 Periapical radiographs

Periapical radiographs obtained 6 (A), 21 (B), 35 (C), and 54 (D) days after the first visit.

complicated anatomical features involved ${ }^{8}$. Herein, we present a case of recurrent gingival abscess identified in a fused tooth composed of the central incisor and a supernumerary tooth.

\section{Case Report}

A 5Y7M-old girl was referred to our clinic by a general dentist for detailed examination of a recurrent gingival abscess in the maxillary left primary central incisor region. An intraoral examination revealed diffuse swelling in the palatal gingival area of the affected tooth (Fig. 1A, B). According to her mother, the affected tooth had received traumatic injuries several times and a gingival abscess was repeatedly identified on the labial side of the affected tooth region. A periapical radiograph taken by the general dentist 1 day prior to visiting our clinic showed that the affected tooth was actually the central incisor and a supernumerary tooth, which had fused and were filled with composite resin at the juncture of the 2 tooth crowns. The mesiodistal lengths of the mesial and distal crowns were $5.2 \mathrm{~mm}$ and $5.0 \mathrm{~mm}$, respectively, both smaller than that of the maxillary 

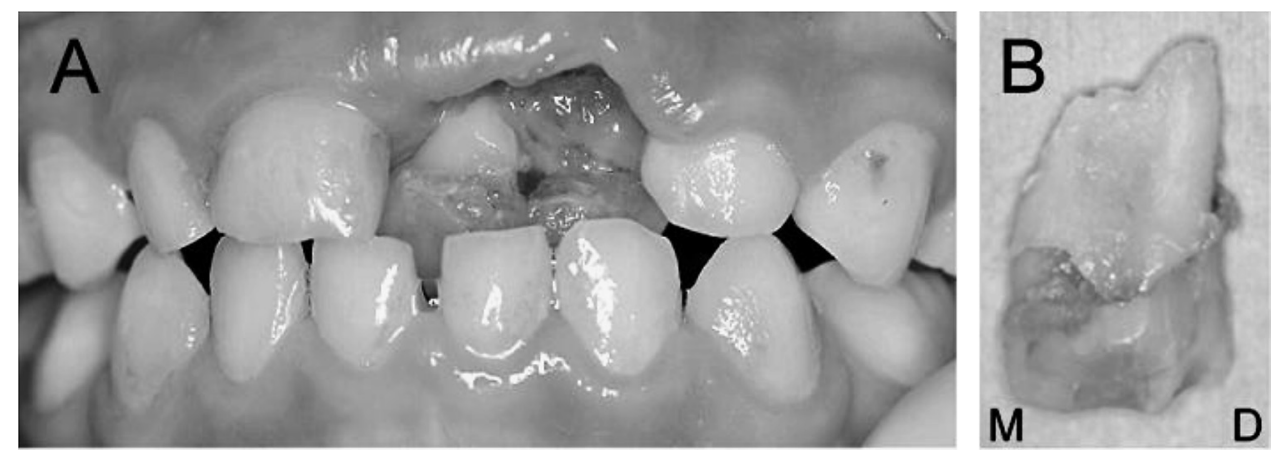

Fig. 3 Affected tooth extracted after a traumatic injury at $6 \mathrm{YOM}$

Frontal view of the affected tooth region (A). Labial view of the 2 fragments of the extracted tooth (B). $\mathrm{M}$ and $\mathrm{D}$ in the photographs indicate the mesial and distal sides, respectively.

right primary central incisor $(7.0 \mathrm{~mm})$. In order to produce a pathway for pus discharge, partial removal of the composite resin was carried out, which caused severe bleeding from the distal side of the crowns, and antibiotics were prescribed for 3 days. At the second visit 6 days later, the abscess lesion was reduced in size, but had not disappeared. As a dental radiograph showed 2 independent root canals in the affected tooth (Fig. 2A), an additional access cavity was produced in the mesial part of the affected tooth to directly reach the mesial part of the root canal. At the third visit (21 days after the first visit), there were no signs or symptoms identified in the affected area, and root canal filling with iodoform-calcium hydroxide paste and restoration with glass ionomer cement were performed (Fig. 2B).

Following those treatments, the affected tooth was restored with composite resin as a single crown, as the patient and her mother preferred restoration to the same as the original tooth. There were no signs or symptoms reported at the fourth (35 days after the first) and fifth (54 days after the first) follow-up visits (Fig. 1C and D). However, radiographic images obtained at those examinations revealed that the paste used for the canal fillings had been adsorbed in the cervical area of the root (Fig. 2C and D).

At $6 \mathrm{YOM}$, the patient received a traumatic injury in the anterior region and the fused tooth was displaced in a palatal direction (Fig. 3A). Since it was difficult to preserve, we decided to extract the tooth (Fig. 3B). Observations of the extracted tooth showed that the root was wide in the mesiodistal direction and the root tip of the mesial canal was slanted toward the palatal side, while the distal one was toward the labial side. The fused tooth

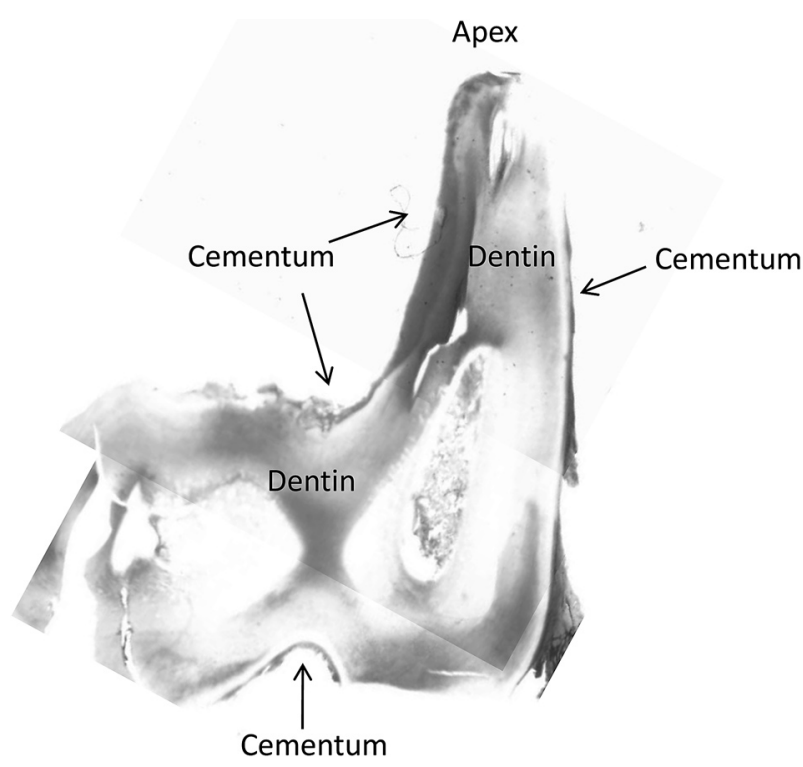

Fig. 4 Histopathological appearance

Shown is a portion of the root of the fused tooth. The root had 2 root canals and dentin was shared.

was decalcified, sliced in a mesiodistal manner, and stained with Hematoxylin-Eosin for a histopathological examination, which revealed that the root had 2 narrow canals, with only dentin found between the 2 root canals (Fig. 4).

\section{Discussion}

The present patient was referred to our clinic by a general dentist for consultation regarding recurrent gingival abscess formation in the area of the maxillary left primary central incisor, which had fused to a supernumerary tooth. The fused tooth had 2 
separate crowns, with each showing a similar size and shape. Removal of composite resin from the central areas of the fused tooth induced severe bleeding from the distal sides of the crowns. A slight gingival abscess lesion remained 6 days later, thus direct exposure of the mesial side of pulp chamber was performed, which enabled the swelling to disappear. Therefore, the recurrent gingival abscess formation in the present case was estimated to be derived from necrosis of the pulp in both teeth.

A radiographic examination showed that the fused tooth had 1 root with 2 separate canals, and canal fillings with iodoform-calcium hydroxide paste was performed. Periapical radiographs later revealed that the paste disappeared from the cervical area to the apex area of the root, suggesting the existence of communication to the oral cavity in the cervical area of the root. The crown of the fused tooth was composed of 2 independent smaller sized tooth crowns. As the patient and her mother wanted to rehabilitate the affected tooth as a single tooth, we restored the affected tooth with a large crown using composite resin. However, we should have chosen to restore with resin as 2 separate crowns, as a leak may have been present in the cervical area of the fused tooth.

Extraction of the fused tooth made it possible to examine its shape and morphology. We observed that it possessed 2 crowns and a single root, including 2 root canals. Histopathological examination revealed that dentin had united the roots of the central incisor and supernumerary tooth, suggesting that 2 tooth germs had been joined by dentin during the root formation period.

Surprisingly, the permanent successor of the fused tooth has a similar size and morphology as the permanent central incisor on the opposite side in periapical radiographs (Fig. 2). Thus, the fused tooth must have been composed of the primary central incisor and a supernumerary primary tooth. In theory, each tooth should have a permanent successor or those would be expected to fuse with each other to form a large fused tooth. In addition, the maxillary left lateral primary incisor next to the fused teeth has no permanent successor. The mesiodistal lengths of the fused primary tooth were $5.2 \mathrm{~mm}$ for the mesial crown and $5.0 \mathrm{~mm}$ for distal crown. If the distal crown is to be a supernumerary tooth, as it was smaller than the mesial crown, the 2 adjacent primary teeth, a maxillary left supernumerary tooth and the lateral incisor, have no permanent successors. Thus, localized failure may occur in this area.

In summary, we treated an uncommon case of fused tooth of the maxillary primary central incisor and a supernumerary primary tooth, with showed repeated gingival abscess formation. The major cause for the repeated lesion may be derived from the necrosis of the both pulp tissues of the affected fused tooth. Root canal treatment that produced an additional access cavity to the mesial part of the fused tooth resulted in temporary success. The fused tooth had 2 tooth crowns and a single root with 2 root canals, each of which should have been treated separately.

\section{References}

1) Braham, R.L.: Developmental anomalies of the dentition: a scientific review. Pediatr Dent 5: 105116, 1995.

2) Schuurs, A.H. and van Loveren, C.: Double teeth: Review of the literature. ASDC J Dent Child 67: 313-325, 2000.

3) Kunimatsu, H., Miyoshi, S., Sato, A. and Shimizu, T.: Bilateral deciduous fused incisors. Jpn J Pediatr Dent 32: 14-20, 1994. (in Japanese).

4) Tsujino, K., Kurosu, M., Katane, T., Mochizuki, K., Yonezu, T. and Yakushiji, M.: Fused deciduous teeth and their relationship with the permanent successors: a study of 182 cases. Jpn J Pediatr Dent 36: 861-866, 1998. (in Japanese).

5) Miyoshi, S., Tanaka, S., Kunimatsu, H., Murakami, Y., Fukami, M. and Fujisawa, S.: An epidemiological study of supernumerary primary teeth in Japanese children: a review of racial differences in the prevalence. Oral Dis 6: 99-102, 2000.

6) Stillwell, K.D. and Coke, J.M.: Bilateral fusion of the maxillary central incisors to supernumerary teeth: report of case. J Am Dent Assoc 112: 62-64, 1986.

7) Crawford, N.L., North, S. and Davidson, L.E.: Double permanent incisor teeth: management of three cases. Dent Update 33: 608-610, 2006.

8) Ballal, S., Sachdeva, G.S. and Kandaswamy. D.: Endodontic management of a fused mandibular second molar and paramolar with the aid of spiral computed tomography: a case report. J Endod 33: 1247-1251, 2007. 